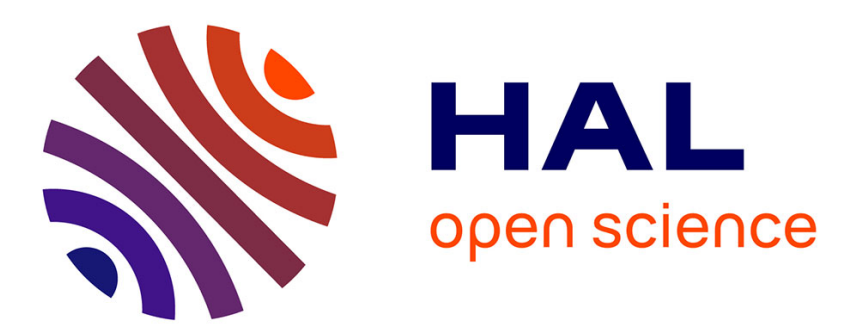

\title{
Multiple myeloma treatment at relapse after autologous stem cell transplantation: a practical analysis
}

\author{
F. Malard, J.L. Harousseau, M. Mohty
}

\section{To cite this version:}

F. Malard, J.L. Harousseau, M. Mohty. Multiple myeloma treatment at relapse after autologous stem cell transplantation: a practical analysis. Cancer Treatment Reviews, 2016, 10.1016/j.ctrv.2016.11.005 . hal-01400036

\section{HAL Id: hal-01400036 \\ https://hal.sorbonne-universite.fr/hal-01400036}

Submitted on 21 Nov 2016

HAL is a multi-disciplinary open access archive for the deposit and dissemination of scientific research documents, whether they are published or not. The documents may come from teaching and research institutions in France or abroad, or from public or private research centers.
L'archive ouverte pluridisciplinaire HAL, est destinée au dépôt et à la diffusion de documents scientifiques de niveau recherche, publiés ou non, émanant des établissements d'enseignement et de recherche français ou étrangers, des laboratoires publics ou privés. 


\section{Multiple myeloma treatment at relapse after autologous stem cell transplantation: a practical analysis}

F Malard ${ }^{1,2,3}$, JL Harousseau ${ }^{4}$ and M Mohty ${ }^{1,2,3}$

1- Department of Haematology, Saint Antoine Hospital, Paris, France 2- INSERM UMRs 938, Paris, France

3- Université Pierre et Marie Curie, Paris, France;

4-Centre Catherine de Sienne, Nantes, France

Running title: Treatment of MM relapse after ASCT

Keywords: multiple myeloma, transplant eligible patients, first relapse, autologous stem cell transplantation, new agents, treatment

Word count: abstract: 166; main text: 4067; table: 1; figure: 0 .

\section{Authors for correspondence:}

Dr Florent Malard; Service d'Hématologie Clinique et de Thérapie Cellulaire, Hôpital Saint Antoine, APHP, Université Pierre et Marie Curie and INSERM, UMRs 938, 184 rue du Faubourg Saint-Antoine, 75012, Paris, France. Phone : +33 149282629 ; email: malardf@yahoo.fr 


\title{
Multiple myeloma treatment at relapse after autologous stem cell transplantation: a practical analysis
}

\begin{abstract}
Over the past decade, significant advances have been made in the field of multiple myeloma. Introduction of the so-called novel agents, proteasome inhibitors (PI) and immunomodulatory drugs (IMiD), and improved supportive care have resulted in significantly better outcome. Standard first line treatment in fit patients include PI and IMiD based induction, high dose melphalan with autologous hematopoietic stem cell transplantation (ASCT) and consolidation/maintenance. However, despite these progresses MM remains incurable for the majority of patients and most patients will relapse. Next generation PI (carfilzomib, ixazomib) and IMiD (pomalidomide) and new therapeutic classes: monoclonal antibody (elotuzumab, daratumumab) and pandeacetylase inhibitors (panobinostat) have been successfully evaluated in relapse multiple myeloma. Some of these new agents are now approved for multiple myeloma treatment at relapse. However choosing the most appropriate treatment at relapse may be difficult. This review sum up the most important studies and provide evidence to choose the most relevant therapeutic strategy for relapse after ASCT, based on disease, patient and previous treatment related parameters.
\end{abstract}

Keywords: multiple myeloma, transplant eligible patients, first relapse, autologous stem cell transplantation, new agents, treatment 


\section{Clinical situation}

The patient is a 60-year-old man with a newly diagnosed multiple myeloma, and no medical history. He is still active, working as a clerk and lives independently with his wife. Baseline assessment shows an IgG lambda multiple myeloma, with a monoclonal IgG protein level of $34 \mathrm{~g} / \mathrm{L}$, hemoglobin $10.3 \mathrm{~g} / \mathrm{dL}$, calcium 2.4 mmol/L, creatinine $82 \mu \mathrm{mol} / \mathrm{L}$, albumin $32 \mathrm{~g} / \mathrm{L}$ and $\beta 2$-microglobulin $4.2 \mathrm{mg} / \mathrm{L}$ (ISS II). The skeletal survey reveals lytic lesions in the skull, the ninth thoracic vertebra and the left humerus, and a bone marrow biopsy shows 45 percent involvement by abnormal plasma cells. There is no $\mathrm{t}(4 ; 14)$, nor del17p by FISH. He is treated with induction bortezomib, lenalidomide and dexamethasone (VRD), and serum protein electrophoresis demonstrates a partial response with $>70 \%$ decrease in monoclonal IgG protein level $(10 \mathrm{~g} / \mathrm{L})$. He then receives high dose melphalan (HDM) with autologous hematopoietic stem cell transplantation (ASCT) and support consolidation with 2 additional cycles of VRD. He did not accept to receive any maintenance therapy after ASCT. Response evaluation at 3 months after ASCT (serum electropheresis, immunofixation and bone marrow aspirate) reveal complete remission. However, the patient is concerned about relapse risk and treatment options. He returns to discuss further management.

\section{Challenges in diagnosis of relapse}

Multiple myeloma (MM), is the second most common hematologic

malignancy[1]. Over the past decade, significant advances have been made in the field of MM. Introduction of the so-called novel agents, proteasome inhibitors (PI) and immunomodulatory drugs (IMiD), and improved supportive care have resulted in significantly better outcome. However, MM remains incurable for the majority of patients. At present, a definition of cure requires a relapse-free interval of at least 10-15 years [2, 3]. After HDM and ASCT, at most 10-15\% of patients fall in this category[3]. Therefore, the large majority of MM patients will relapse.

According to the International Myeloma Working Group criteria, progressive disease in MM is defined as an increase of $25 \%$ from nadir in the serum (with a minimum value of $0.5 \mathrm{~g} / \mathrm{dL}$ ) or the urine $\mathrm{M}$-component (with a minimum value 
of $200 \mathrm{mg} / 24 \mathrm{H}$ ), or an increase in the difference between involved and noninvolved free-light chain immunoglobulin $>10 \mathrm{mg} / \mathrm{dL}[4]$. In patients who lack measurable paraprotein level, progression is defined by an increase in bone marrow plasma cells $\geq 10 \%$, development of new/increasing size of bone/soft tissue lesion, or unexplained serum calcium > $2.65 \mathrm{mmol} / \mathrm{L}[4]$. Refractory relapse MM is defined as disease progression on therapy or within 60 days of the last treatment in patients who had achieved a minimal response[5], while patients who never achieved a such response and progress while on therapy are defined as "primary refractory"[5]. By definition, a clinical relapse requires direct indicators of increasing disease and end organ dysfunction (CRAB features: symptoms of hypercalcemia, renal insufficiency, anemia and bone disease), in addition to biochemical progression[4].

Significant advances have been made in the field of multiple myeloma over the past years and several therapeutic options are available at relapse.

\section{Summary of current practices}

Thalidomide (THAL), bortezomib (BTZ) and lenalidomide (LEN) have been extensively studied in relapse or refractory relapse MM[6].

Thalidomide. A systematic review of phase II trials of combination of THAL and dexamethasone (DEX) reported an overall response rate [overall response rate (ORR): complete remission (CR) + partial remission (PR)] of 46\% in patients not previously exposed to an IMiD[7]. A phase III prospective study compare THAL + DEX to BTZ + DEX in 131 patients with refractory relapse MM and not previously exposed to an IMiD[8]. Median progression-free survival (PFS) and ORR were similar between the THAL group (respectively, 9.0 months and 55\%) and the BTZ group (respectively, 7.2 months and 63\%). This study suggest that THAL + DEX have an efficacy comparable to BTZ + DEX in refractory relapse MM patients not previously exposed to IMiDs. However, disappointing results have been reported using THAL at relapse in patients initially treated with IMiDs[9]. Therefore, given that most patients do receive an IMiD as part of first-line treatment, the role of THAL in relapsed MM is very limited. THAL + DEX remains a valid, cost-effective, option in patients not previously exposed to IMiDs. 
Bortezomib. A phase II study evaluated retreatment with BTZ, in a cohort of 130 patients responding for at least 6 months to this drug[10]. Such retreatment, without or with DEX, was associated with an ORR of 40\%[10]. However, further improvement can be obtained by adding a third drug to BTZ + DEX. This can either be an alkylating agent (cyclophosphamide (Cy)[11, 12], bendamustine[13], melphalan[14]), an anthracycline (pegylated doxorubicin[15]), an IMiD (THAL[16], LEN[17]), or a histone deacetylase inhibitor (panobinostat[18], vorinostat[19]). These combinations lead to higher ORR, from 55\% to 87\%. Although subcutaneous administration of BTZ significantly decreases the incidence of peripheral neuropathy[20], retreatment with BTZ must be avoided in patients with persistent peripheral neuropathy (Grade $\geq 2$ ).

Lenalidomide. Two randomized phase 3 trials have evaluated LEN + DEX versus DEX alone in relapsed or refractory relapse MM[21, 22] (Table 1). The superiority of the LEN + DEX combination was confirmed at a median follow-up of 48 months in a pooled update of both trials[23]. The median PFS was 11.1 months and the ORR was $60.6 \%$ in the LEN + DEX group[23]. Furthermore, there was a significant benefit in overall survival in patients treated with LEN + DEX (median of 38.0 versus 31.6 months, $\mathrm{p}=0.045$ ), despite the fact that $47.6 \%$ of those who were randomized to DEX+ placebo ended up receiving a LEN-based treatment after disease progression or study unblinding[23]. These studies led to approval of the drug, and the combination LEN + DEX is considered a standard for relapsed MM. It has been the backbone for several studies evaluating new agents as part of a three-drug regimen in relapse MM.

Carfilzomib. Stewart et al. reported the results of the randomized phase 3 ASPIRE trial, comparing the combination LEN, DEX and carfilzomib (CFZ) with that of LEN + DEX alone[24]. CFZ is a second-generation epoxyketone proteasome inhibitor, that binds selectively and irreversibly to the constitutive proteasome and immunoproteasome[24]. A series of 792 patients with relapsed MM after a median of two lines of treatment were randomized to receive one of the two combinations. The PFS was significantly improved with CFZ (median, 26.3 months, vs. 17.6 months in the control group; $P=0.0001)$. This improved PFS in the CFZ group was observed among patients previously treated with BTZ 
or LEN, and in patients with high-risk cytogenetic (defined by the presence of $\mathrm{t}(4 ; 14), \mathrm{t}(14 ; 16)$ or deletion $17 \mathrm{p})$. The ORR were $87.1 \%$ and $66.7 \%$, in the LEN $+\mathrm{DEX}+\mathrm{CFZ}$ and the LEN + DEX groups, respectively $(P<0.0001)$. Of note, there were no differences in adverse events of grade 3 or higher, and patients in the CFZ group reported better quality of life. These impressive results led to the approval by the Food and Drug Administration (FDA) and the European Medicines Agency (EMA) of CFZ in combination with LEN and DEX in patients with relapsed MM who have received 1 to 3 prior lines of treatment for the FDA or at least one previous line of treatment for the EMA. CFZ promotes a better proteasome inhibition than BTZ[25], is effective in BTZ-refractory patients[24, $26]$, and is associated with a lower rate of peripheral neuropathy, even when compared to subcutaneous BTZ[26]. Dimopoulos et al. reported recently the results of the randomized phase 3 ENDEAVOR trial comparing the combinations $\mathrm{CFZ}+\mathrm{DEX}$ and BTZ + DEX in relapse or refractory relapse MM[27]. The median PFS was 18.7 months in the CFZ group versus 9.4 months in the BTZ group $(P<0.0001)$. The ORR were $77 \%$ and $63 \%$, in the $\mathrm{CFZ}$ and the BTZ groups, respectively $(P<0.0001)$. The number of patients who had grade $\geq 2$ peripheral neuropathy, was significantly higher in the BTZ group (32\% versus $6 \%$ in the CFZ group; $P<0.0001)$. However, we must keep in mind that, while BTZ can be administered subcutaneously, CFZ administration is strictly intravenous and requires 6 infusion visits per 28-day cycle[24]. In addition, proteasome inhibition has the potential of significant cardiac toxicity via the accumulation of intracellular protein aggregate[28]. The ENDEAVOR trial reported a higher incidence of cardiac failure grade $\geq 3$ in the CFZ group (4.3\% versus $1.7 \%$ in the BTZ group)[27]. A pooled analysis of CFZ safety in 526 patients with advanced MM treated as part of clinical trial, reported cardiac failure in $7.2 \%$ of patients[26]. Therefore, particular attention must be paid to patients with history of cardiac failure or ongoing cardiac failure.

Ixazomib. Another next generation proteasome inhibitor, Ixazomib (IXA), has been evaluated in combination with LEN and DEX in the randomized phase 3 TOURMALINE-MM1 trial[29]. IXA is a reversible proteasome inhibitor, which has the advantage of being orally administered. A total of 722 patients with refractory relapse MM were randomized to receive LEN, DEX and IXA or LEN and 
DEX alone. The PFS was significantly improved with IXA (median, 20.6 months, vs. 14.7 months in the control group; $P=0.012$ ). The ORR was also improved in the IXA group, being $78 \%$ vs. $72 \%$ in the control group ( $\mathrm{p}=0.035)$. No increase in overall toxicity was reported in the IXA group. Although addition of IXA to LEN and DEX resulted in a higher rate of peripheral neuropathy (27\% versus $22 \%$ in the control group)[29], the incidence of grade 3 events was only $2 \%$ compared to $3 \%$ in patients who received CFZ[24] and 6\% in those treated with subcutaneous BTZ[20]. This study led to the approval by the FDA of IXA in combination with LEN and DEX in relapsed MM patients who had received at least one prior therapy. IXA also received a positive opinion of the Committee for Medicinal Products for Human Use of EMA on September 16, 2016. Of note, IXA is the first in class oral proteasome inhibitor and the LEN + DEX + IXA triplet offers the advantage of being fully orally administered.

Monoclonal antibodies. Several studies evaluated triplet combinations including a monoclonal antibody, elotuzumab (ELO) or daratumumab (DARA). ELO is an immunostimulatory monoclonal antibody targeting signaling lymphocytic activation molecule F7 (SLAMF7), a glycoprotein expressed on myeloma and natural killer cells but not on normal tissues, that enables selective killing of myeloma cell with minimal effect on healthy tissue[30]. The randomized phase 3 ELOQUENT-2 trial evaluated ELO in combination with LEN and DEX, as compared with LEN and DEX alone in 646 patients with refractory relapse MM[31]. The median PFS in the ELO group was 19.4 versus 14.9 months in the control group $(P<0.001)$. The ORR was $79 \%$ versus $66 \%$ in the control group $(P<0,001)$. There was no difference in the serious adverse event incidence between the two groups. These findings led to the approval by the FDA and the EMA of ELO in combination with LEN and DEX in patients with relapsed MM who had received $\geq 1$ prior lines of treatment (up to 3 for the FDA). ELO has also been evaluated in combination with a PI and steroids. A randomized phase II trial compared the combination of ELO, BTZ and DEX versus BTZ and DEX alone in 152 patients with refractory relapse MM[32]. The median PFS in the ELO group was 9.7 months, versus 6.9 months in the control group $(P=0.09)$, with corresponding ORR of $66 \%$ versus $63 \%$. This study suggests that the addition of 
ELO to BTZ and DEX may improve PFS, however confirmatory studies are necessary.

The second monoclonal antibody with an advanced clinical development, DARA, targets CD38. A phase 1/2 trial evaluates DARA in combination with LEN and DEX[33]. The first phase was a dose escalation study to identify DARA maximum tolerated dose. The second part was a cohort expansion to evaluate DARA, administered at the maximum tolerated dose $(16 \mathrm{mg} / \mathrm{kg})$, in combination with LEN and DEX. In the 32 patients treated in the expansion cohort, the ORR was 81\%, including 6 (19\%) PR and 9 (28\%) very good partial responses, 3 (9\%) CR and 8 (25\%) stringent CR. These impressive results and the good tolerance of the combination, lead to a prospective randomized phase 3 study POLLUX, evaluating the addition of DARA to the combination of LEN and DEX versus LEN and DEX alone[34]. 569 patients with refractory relapse MM were included. With a median follow-up of 13.5 months, the median PFS was not yet reached in the DARA group, versus 18.4 months in the control group $(P<0.001)$. A significantly higher ORR was observed in the DARA group than in the control group (93\% versus $76 \%, P<0.001$ ), as was a higher rate of $C R$ or better ( $43 \%$ versus $19 \%$, $P<0.001)$. Furthermore, in the DARA group, $22 \%$ of patients had results below the threshold for minimal residual disease as compared with $5 \%$ of those in the control group $(P<0.001)$. No increase in overall toxicity was reported in the DARA group beside infusion reactions related to DARA. Infusion reactions were reported in $48 \%$ of patients receiving DARA and were mostly grade $1 / 2$ with only $5 \%$ experiencing grade 3 .

DARA efficacy has also been evaluated in combination with BTZ and DEX in the prospective randomized phase 3 study CASTOR[35]. A total of 498 patients with refractory relapse MM were randomized to receive DARA, BTZ and DEX or BTZ and DEX alone. With a median follow-up of 7.4 months, the median PFS was not yet reached in the DARA group, versus 7.2 months in the control group $(P<0.001)$. The ORR was also improved in the DARA group, being 79\% versus $66 \%$ in the control group $(P<0.001)$, similarly the rate of $\mathrm{CR}$ or better was significantly improved in the DARA group compared to the control group (19\% versus 9\%; $P=0.001$ ) No increase in overall toxicity was reported in the DARA group. Although in the DARA group, $45 \%$ of patients presented DARA infusion- 
related reaction, with a majority of grade $1 / 2$ reaction and $9 \%$ of grade 3 reaction.

Currently, DARA monotherapy is approved by the FDA in refractory relapse MM patients who have received at least 3 prior lines of therapy, including a proteasome inhibitor and an IMiD agent, or who are double refractory to a proteasome inhibitor and an IMiD agent, based on the phase 2 MMY2002 and the phase 1/2 GEN501 monotherapy studies[36, 37]. DARA monotherapy is also approved by the EMA in refractory relapse MM patients whose prior therapy, included a proteasome inhibitor and an IMiD agent and who have demonstrated desease progression on the last treatment.

Pan-deacetylase inhibitors. Pan-deacetylase inhibitors, which are epigenetic modulators, have emerged as a novel class of anti-myeloma drug. A randomized phase-3 trial, PANORAMA-1, evaluated panobinostat (PAN), a pan-deacetylase inhibitor, in combination with BTZ and DEX, versus placebo, BTZ and DEX, in relapsed or refractory relapse MM[18]. Patients were in an early relapse setting (1 to 3 previous lines of treatment). The median PFS was significantly higher in the PAN group: 11.99 versus 8.08 months in the control group $(P<0.0001)$. The ORR was not significantly different between the PAN, $60.7 \%$ and the control group, $54.6 \%(P=0.09)$. PAN, in combination with BTZ and DEX, is approved by the FDA and the EMA for patients having received $\geq 2$ lines of treatment, including BTZ and an IMiD. However, in the PANORAMA-1 trial, the association $\mathrm{PAN}+\mathrm{BTZ}+\mathrm{DEX}$ was associated with an increased incidence of grade 3 thrombocytopenia, diarrhea, asthenia and peripheral neuropathy. Therefore, while use of SC instead of IV BTZ may improve the safety profile, this combination must be handled cautiously.

Another histone deacetylase inhibitor, vorinostat (VOR), has been evaluated in early relapse MM[19]. The phase-3 VANTAGE-088 trial randomized patients to VOR + BTZ or BTZ alone[19]. The median PFS was significantly higher in the VOR group, 7.63 versus 6.83 months in the control group $(P=0.01)$. However, with a difference of less than a month, the clinical relevance of this combination is small and limited[19].

Pomalidomide. Pomalidomide (POM), a third generation IMiD, have been evaluated in a randomized phase 3 study in patients with relapsed or refractory 
relapse MM, previous BTZ and LEN failure and adequate prior alkylating therapy [38]. Patients were randomized to receive either POM and low-dose DEX or high-dose DEX alone. In those heavily pretreated patients (median of 5 previous lines of treatment), the median PFS was significantly higher in the POM group: 4.0 versus 1.9 months in the control group. An phase $3 \mathrm{~b}$ study, confirms a median PFS of 4.6 months on a similar population of 682 patients[39]. These results were the basis for POM approval by the FDA and the EMA (in combination with low-dose DEX in Europe) for patients who had received $\geq 2$ previous lines of treatment, including LEN and DEX, and progressive under previous therapy. POM was also evaluated in combination with BTZ + DEX and $\mathrm{CFZ}+\mathrm{DEX}$ as part of a phase $1 / 2$ clinical trial in refractory relapse $\mathrm{MM}$, paving the way for the use of a triplet regimen including POM at first relapse.

Cyclophosphamide. Cyclophosphamide is an alkylating agents used orally or intravenously and usually well tolerated in MM. Several triplet regimens including Cy in combination with THAL, LEN or BTZ in refractory relapse MM have been reported. Kyriakou et al. reported an ORR of 79\% and a 2-years PFS of $34 \%$ using the combination of Cy, THAL and DEX in a phase I/II study[40]. Two phases I/II study evaluate the combination of Cy, LEN and either DEX[41] or prednisone[42] in refractory relapse MM with an ORR of $81 \%$ and $94 \%$ respectively. 2-years PFS was 56\% with the combination of Cy + LEN + DEX, and median PFS was 16.4 months using the combination of Cy + LEN + prednisone. Kropff et al. performed a phase II study evaluating the combination of Cy, BTZ and DEX for refractory relapse MM[12]. This combination was associated with an ORR of $22 \%$ and a median PFS of 12 months. Similarly, the combination of Cy, BTZ and prednisone have been evaluated in a phase I/II study with an ORR of $89 \%$ at the highest dose level and a 1-year PFS of 83\%[11]. Overall, triplet regimens combining IMiD or BTZ with steroids and Cy appear to be an effective strategy, particularly cost-effective compared to the new agents based triplet regimens.

Second ASCT. The role of second HDM-ASCT in relapsed MM has also been investigated. A randomized phase 3 study, included patients with first progressive or relapsed disease at least 18 months after a previous ASCT[43]. All eligible patients received BTZ, doxorubicin and DEX induction therapy, and 
peripheral blood stem cell mobilization and harvesting. Patients with adequate stem-cell harvest were randomized to HDM $200 \mathrm{mg} / \mathrm{m}^{2}$ plus salvage ASCT or oral Cy. The median PFS was significantly longer in the ASCT group (19 compared to 11 months in the Cy group; $\mathrm{p}<0.0001$ ] [43]. Furthermore, a recently published update, confirm that ASCT improve PFS and show an advantage in OS in the ASCT group: median OS was significantly higher in the ASCT group, 67 months, versus 35 months in the Cy group $(P=0.0169)$ [44]. However, the results of this study must be interpreted with caution. Oral Cy is not a standard treatment for first relapse after HDM-ASCT, in contrast to the highly effective combination therapies including PI and IMiD. Therefore, a decision to proceed to a second HDM-ASCT must be carefully weighed against a combination of new agents. A second HDM-ASCT should probably be considered only in fit, transplant-eligible patients, with a long PFS after the first HDM-ASCT $(\geq 18$ months)[45].

Allogeneic transplantation. The feasibility of allogeneic hematopoietic stem cell transplantation (allo-HCT) with a reduced intensity conditioning regimen (RIC) in relapsed MM has been demonstrated in several studies[46-48]. However, RIC allo-HCT is still associated with high non-relapse mortality, around $20-25 \%$ at 1 year, and a high relapse incidence, leading to a 2 year PFS of 26-38\%[46-48]. Therefore, allo-HCT should not be performed routinely outside clinical trials; it may be an option in very few selected patients, particularly those with high-risk disease[45].

\section{General management approaches}

The immediate aim of treatment at relapse is disease control to treat/prevent CRAB symptoms, relieve the patient's symptoms and avoid end organ damage. The impact of the depth of the response on survival is still controversial in the relapse setting[6]. However, growing amount of data suggest a relationship between the two parameters, demonstrating that a better quality of response may be associated with an improved outcome, even beyond first line treatment[6, 49-51]. Therefore, patients with good performance status at relapse should receive therapy designed to achieve the deepest possible response in order to improve survival[6]. 
At relapse, treatment choice must be individualized in order to identify the treatment with the best efficacy versus toxicity balance for each patient. Overall, disease-related, patient-related, and previous treatment-related parameters must be taken into account for the treatment selection in MM at relapse.

Disease-related parameters. The clinical course of MM is very different from one patient to another. In patients with symptomatic relapse with CRAB features, threatening or not, treatment is mandatory and cannot be delayed. In contrast, in patients with asymptomatic relapse, the decision to treat depends on the paraprotein kinetic increase. Those with a doubling of the M-component within 2 months should be treated[5]. For the remainder, a careful watch and wait policy every 1-3 months is recommended[5].

In addition, the therapeutic strategy will be different in patients with a standardrisk from that for high-risk MM. The latter must receive immediate combination therapy at relapse. Based on the International Myeloma Working Group (IMWG) recommendations for treatment of high-risk MM, BTZ and CFZ treatment improve CR, PFS and OS in $t(4 ; 14)$ and del(17p) whereas LEN may be associated with improved PFS in $(4 ; 14)$ and $\operatorname{del}(17 \mathrm{p})$ and POM show promising results in $\operatorname{del}(17 p)[52]$. Therefore, we suggest to use a treatment combining a PI and an IMiD in high-risk cytogenetic. Monoclonal antibodies may be used in those patients, however data are required to know they overcome the bad prognosis of high-risk cytogenetic. For patients with threatening CRAB features/aggressive relapse, attention must be paid to the kinetic of response expected, in order to favor treatment with a quick expected response. Response to new generation PI, CFZ and IXA, in combination with LEN + DEX, is quite quick with a median time to first response of one month[24, 27]. For monoclonal antibody, kinetic of response to DARA monotherapy is similar to that of PI, about a month[36, 37], while response to ELO, in combination with LEN + DEX, is rather slow, with a median time to first response of 50 days[53]. Median time to first response using pan-deacetylase inhibitors in combination with BTZ +/- DEX is also longer compare to PI, ranging from 36 days using VOR[19] to 1.5 months with PAN[18, 54]. Finally response to POM + DEX is also rather slow with a median time to first response of 1.9 months. [55] Whenever possible, these patients should be included in a clinical trial evaluating novel agents. High-risk MM are patients 
with extramedullary disease or plasma cell leukemia, adverse cytogenetics (del17p or $\mathrm{t}(4 ; 14)$ ), a high-risk gene expression profile, or an International Staging System (ISS) 3 (low albumin and high $\beta 2$-microglobulon level) and high lactate dehydrogenase[56-58].

Patient-related parameters. Patients' age and performance status must be considered before choosing the best treatment. However, in the setting of the first relapse after ASCT, most patients are usually in good general condition, and frailty is not an issue. More important in these patients, is the evaluation of renal function and pre-existing toxicities. Renal insufficiency at relapse may be caused by either disease progression or associated predisposition conditions. All PIs (BTZ[59], CFZ[26] and IXA[60]) can be used without dose adjustment in patients with impaired renal function. Among IMiDs, while LEN requires dose adjustments, both THAL[61] and POM[62] do not. The histone deacetylase inhibitors PAN and VOR can be used at their standard doses and may be associated with a renoprotective effect[63, 64]. Regarding monoclonal antibodies, ELO can be safely used with no dose adjustment, including in patients with end-stage renal disease[65]. We expected that DARA tolerance will be similar, DARA renal safety has been shown only in patients with moderate renal impairment [34-36]. For alkylating agents, cyclophosphamide does not require dose adjustment, but melphalan does[66]. While ASCT is feasible in MM with renal insufficiency, usually when using a reduced dose of melphalan (140 $\mathrm{mg} / \mathrm{m}^{2}$ )[67], data evaluating second ASCT at relapse in patients with renal insufficiency are scarce. Given the increased risk of transplant-related mortality for patient with renal insufficiency compared to those without[67], the decision to proceed to a second ASCT in these patients at relapse must be carefully weighted. Combination therapy is feasible in patients with renal insufficiency, provided any dose adjustments are respected. The International Myeloma Working Group has recently published recommendations for the management and dose adjustment of MM related renal impairment[66].

Previous treatment-related parameters. The patients' tolerability of a previous treatment, in particular pre-existing toxicity, must be carefully checked. In patients with grade $\geq 2$ peripheral neuropathy, BTZ should be avoided and replaced by a second generation PI. Retreatment with IMiDs in patients with a 
severe history of thromboembolic events must be very cautious and associated with full anticoagulation therapy $[68,69]$. In patients who experienced severe myelosuppression during the first line, use of most myelosuppressing agents, such as alkylating drugs, must be avoided. For combination therapy design, lack of overlapping toxicities, such as peripheral neuropathy and myelosuppression, must be favored.

The depth of response and remission duration after the first line of treatment must be evaluated. Patients who relapse within 12 months after ASCT have a poor outcome[70], and should receive combination therapy (triple combination whenever available) including novel agents, if possible within a clinical trial. In contrast, in patients with a longer duration of response, re-treatment with prior therapies is feasible. However, in the setting of the first relapse after ASCT, retreatment should be done as part of combination therapy, in order to improve the quality of the response. A second ASCT could be considered in patients with a PFS $\geq 18$ months[45].

Finally, treatment approval by the FDA or EMA must also be taken into consideration. In this regard, combinations therapy based on the pandeacetylase inhibitor PAN or POM are only approved for patients having received $\geq 2$ lines of treatment and therefore are not the first option for treatment at first relapse after ASCT.

The patient treated in our case vignette ultimately presented a clinical relapse 2 years latter, with a high disease burden. He immediately received a second line treatment combining re-treatment with LEN in association with a second generation PI, CFZ and DEX, followed by a second ASCT. The patient finally achieved a second complete remission. 


\section{Funding:}

The work was supported by a grant from the "Association for Training,

Education and Research in Hematology, Immunology and Transplantation"

(ATERHIT), no grant number is applicable.

\section{Conflict of interest disclosure:}

MM received lectures honoraria and/or research support from Janssen, Celgene,

Amgen, Takeda, Novartis and Bristol-Myers whose products are discussed in this work.

FM and JLH have declared no conflicts of interest.

\section{Authorship}

FM, JLH and MM designed the manuscript, analyzed the literature, and wrote the manuscript 


\section{References}

1. Siegel RL, Miller KD, Jemal A. Cancer statistics, 2015. CA Cancer J Clin. 2015;65:5-29.

2. Barlogie B, Mitchell A, van Rhee F, Epstein J, Morgan GJ, Crowley J. Curing myeloma at last: defining criteria and providing the evidence. Blood. 2014;124:3043-51.

3. Martinez-Lopez J, Blade J, Mateos MV, Grande C, Alegre A, Garcia-Larana J, et al. Long-term prognostic significance of response in multiple myeloma after stem cell transplantation. Blood. 2011;118:529-34.

4. Durie BG, Harousseau JL, Miguel JS, Blade J, Barlogie B, Anderson K, et al. International uniform response criteria for multiple myeloma. Leukemia. 2006;20:1467-73.

5. Rajkumar SV, Harousseau JL, Durie B, Anderson KC, Dimopoulos M, Kyle R, et al. Consensus recommendations for the uniform reporting of clinical trials: report of the International Myeloma Workshop Consensus Panel 1. Blood. 2011;117:4691-5.

6. Mohty B, El-Cheikh J, Yakoub-Agha I, Avet-Loiseau H, Moreau P, Mohty M. Treatment strategies in relapsed and refractory multiple myeloma: a focus on drug sequencing and 'retreatment' approaches in the era of novel agents. Leukemia. 2012;26:73-85.

7. von Lilienfeld-Toal M, Hahn-Ast C, Furkert K, Hoffmann F, Naumann R, Bargou $\mathrm{R}$, et al. A systematic review of phase II trials of thalidomide/dexamethasone combination therapy in patients with relapsed or refractory multiple myeloma. Eur J Haematol. 2008;81:247-52.

8. Hjorth M, Hjertner O, Knudsen LM, Gulbrandsen N, Holmberg E, Pedersen PT, et al. Thalidomide and dexamethasone vs. bortezomib and dexamethasone for melphalan refractory myeloma: a randomized study. Eur J Haematol. 2012;88:485-96.

9. Madan S, Lacy MQ, Dispenzieri A, Gertz MA, Buadi F, Hayman SR, et al. Efficacy of retreatment with immunomodulatory drugs (IMiDs) in patients receiving IMiDs for initial therapy of newly diagnosed multiple myeloma. Blood. 2011;118:1763-5.

10. Petrucci MT, Giraldo P, Corradini P, Teixeira A, Dimopoulos MA, Blau IW, et al. A prospective, international phase 2 study of bortezomib retreatment in patients with relapsed multiple myeloma. Br J Haematol. 2013;160:649-59. 11. Reece DE, Rodriguez GP, Chen C, Trudel S, Kukreti V, Mikhael J, et al. Phase III trial of bortezomib plus oral cyclophosphamide and prednisone in relapsed and refractory multiple myeloma. J Clin Oncol. 2008;26:4777-83.

12. Kropff M, Bisping G, Schuck E, Liebisch P, Lang N, Hentrich M, et al.

Bortezomib in combination with intermediate-dose dexamethasone and continuous low-dose oral cyclophosphamide for relapsed multiple myeloma. $\mathrm{Br} \mathrm{J}$ Haematol. 2007;138:330-7.

13. Offidani M, Corvatta L, Maracci L, Liberati AM, Ballanti S, Attolico I, et al. Efficacy and tolerability of bendamustine, bortezomib and dexamethasone in patients with relapsed-refractory multiple myeloma: a phase II study. Blood Cancer J. 2013;3:e162. 
14. Popat R, Oakervee H, Williams C, Cook M, Craddock C, Basu S, et al. Bortezomib, low-dose intravenous melphalan, and dexamethasone for patients with relapsed multiple myeloma. Br J Haematol. 2009;144:887-94. 15. Orlowski RZ, Nagler A, Sonneveld P, Blade J, Hajek R, Spencer A, et al. Randomized phase III study of pegylated liposomal doxorubicin plus bortezomib compared with bortezomib alone in relapsed or refractory multiple myeloma: combination therapy improves time to progression. J Clin Oncol. 2007;25:3892901.

16. Garderet L, Iacobelli S, Moreau P, Dib M, Lafon I, Niederwieser D, et al. Superiority of the triple combination of bortezomib-thalidomide-dexamethasone over the dual combination of thalidomide-dexamethasone in patients with multiple myeloma progressing or relapsing after autologous transplantation: the MMVAR/IFM 2005-04 Randomized Phase III Trial from the Chronic Leukemia Working Party of the European Group for Blood and Marrow Transplantation. J Clin Oncol. 2012;30:2475-82.

17. Richardson PG, Xie W, Jagannath S, Jakubowiak A, Lonial S, Raje NS, et al. A phase 2 trial of lenalidomide, bortezomib, and dexamethasone in patients with relapsed and relapsed/refractory myeloma. Blood. 2014;123:1461-9.

18. San-Miguel JF, Hungria VT, Yoon SS, Beksac M, Dimopoulos MA, Elghandour A, et al. Panobinostat plus bortezomib and dexamethasone versus placebo plus bortezomib and dexamethasone in patients with relapsed or relapsed and refractory multiple myeloma: a multicentre, randomised, double-blind phase 3 trial. Lancet Oncol. 2014;15:1195-206.

19. Dimopoulos M, Siegel DS, Lonial S, Qi J, Hajek R, Facon T, et al. Vorinostat or placebo in combination with bortezomib in patients with multiple myeloma (VANTAGE 088): a multicentre, randomised, double-blind study. Lancet Oncol. 2013;14:1129-40.

20. Moreau P, Pylypenko H, Grosicki S, Karamanesht I, Leleu X, Grishunina M, et al. Subcutaneous versus intravenous administration of bortezomib in patients with relapsed multiple myeloma: a randomised, phase 3 , non-inferiority study. Lancet Oncol. 2011;12:431-40.

21. Dimopoulos M, Spencer A, Attal M, Prince HM, Harousseau JL, Dmoszynska A, et al. Lenalidomide plus dexamethasone for relapsed or refractory multiple myeloma. N Engl J Med. 2007;357:2123-32.

22. Weber DM, Chen C, Niesvizky R, Wang M, Belch A, Stadtmauer EA, et al. Lenalidomide plus dexamethasone for relapsed multiple myeloma in North America. N Engl J Med. 2007;357:2133-42.

23. Dimopoulos MA, Chen C, Spencer A, Niesvizky R, Attal M, Stadtmauer EA, et al. Long-term follow-up on overall survival from the MM-009 and MM-010 phase III trials of lenalidomide plus dexamethasone in patients with relapsed or refractory multiple myeloma. Leukemia. 2009;23:2147-52.

24. Stewart AK, Rajkumar SV, Dimopoulos MA, Masszi T, Spicka I, Oriol A, et al. Carfilzomib, lenalidomide, and dexamethasone for relapsed multiple myeloma. $\mathrm{N}$ Engl J Med. 2015;372:142-52.

25. Kuhn DJ, Chen Q, Voorhees PM, Strader JS, Shenk KD, Sun CM, et al. Potent activity of carfilzomib, a novel, irreversible inhibitor of the ubiquitin-proteasome pathway, against preclinical models of multiple myeloma. Blood. 2007;110:328190 . 
26. Siegel D, Martin T, Nooka A, Harvey RD, Vij R, Niesvizky R, et al. Integrated safety profile of single-agent carfilzomib: experience from 526 patients enrolled in 4 phase II clinical studies. Haematologica. 2013;98:1753-61.

27. Dimopoulos MA, Moreau P, Palumbo A, Joshua D, Pour L, Hajek R, et al. Carfilzomib and dexamethasone versus bortezomib and dexamethasone for patients with relapsed or refractory multiple myeloma (ENDEAVOR): a randomised, phase 3, open-label, multicentre study. Lancet Oncol. 2016;17:2738.

28. Grandin EW, Ky B, Cornell RF, Carver J, Lenihan DJ. Patterns of cardiac toxicity associated with irreversible proteasome inhibition in the treatment of multiple myeloma. J Card Fail. 2015;21:138-44.

29. Moreau P, Masszi T, Grzasko N, Bahlis NJ, Hansson M, Pour L, et al. Oral Ixazomib, Lenalidomide, and Dexamethasone for Multiple Myeloma. N Engl J Med. 2016;374:1621-34.

30. Hsi ED, Steinle R, Balasa B, Szmania S, Draksharapu A, Shum BP, et al. CS1, a potential new therapeutic antibody target for the treatment of multiple myeloma. Clin Cancer Res. 2008;14:2775-84.

31. Lonial S, Dimopoulos M, Palumbo A, White D, Grosicki S, Spicka I, et al. Elotuzumab Therapy for Relapsed or Refractory Multiple Myeloma. N Engl J Med. 2015;373:621-31.

32. Jakubowiak A, Offidani M, Pegourie B, De La Rubia J, Garderet L, Laribi K, et al. Randomized phase 2 study: elotuzumab plus bortezomib/dexamethasone vs bortezomib/dexamethasone for relapsed/refractory MM. Blood. 2016;127:283340 .

33. Plesner T, Arkenau HT, Gimsing P, Krejcik J, Lemech C, Minnema MC, et al. Phase $1 / 2$ study of daratumumab, lenalidomide, and dexamethasone for relapsed multiple myeloma. Blood. 2016.

34. Dimopoulos MA, Oriol A, Nahi H, San-Miguel J, Bahlis NJ, Usmani SZ, et al. Daratumumab, Lenalidomide, and Dexamethasone for Multiple Myeloma. N Engl J Med. 2016;375:1319-31.

35. Palumbo A, Chanan-Khan A, Weisel K, Nooka AK, Masszi T, Beksac M, et al. Daratumumab, Bortezomib, and Dexamethasone for Multiple Myeloma. N Engl J Med. 2016;375:754-66.

36. Lonial S, Weiss BM, Usmani SZ, Singhal S, Chari A, Bahlis NJ, et al. Daratumumab monotherapy in patients with treatment-refractory multiple myeloma (SIRIUS): an open-label, randomised, phase 2 trial. Lancet. 2016;387:1551-60.

37. Lokhorst HM, Plesner T, Laubach JP, Nahi H, Gimsing P, Hansson M, et al. Targeting CD38 with Daratumumab Monotherapy in Multiple Myeloma. N Engl J Med. 2015;373:1207-19.

38. San Miguel J, Weisel K, Moreau P, Lacy M, Song K, Delforge M, et al. Pomalidomide plus low-dose dexamethasone versus high-dose dexamethasone alone for patients with relapsed and refractory multiple myeloma (MM-003): a randomised, open-label, phase 3 trial. Lancet Oncol. 2013;14:1055-66. 39. Dimopoulos MA, Palumbo A, Corradini P, Cavo M, Delforge M, Di Raimondo F, et al. Safety and efficacy of pomalidomide plus low-dose dexamethasone in STRATUS (MM-010): a phase $3 \mathrm{~b}$ study in refractory multiple myeloma. Blood. 2016;128:497-503. 
40. Kyriakou C, Thomson K, D'Sa S, Flory A, Hanslip J, Goldstone AH, et al. Lowdose thalidomide in combination with oral weekly cyclophosphamide and pulsed dexamethasone is a well tolerated and effective regimen in patients with relapsed and refractory multiple myeloma. Br J Haematol. 2005;129:763-70. 41. Schey SA, Morgan GJ, Ramasamy K, Hazel B, Ladon D, Corderoy S, et al. The addition of cyclophosphamide to lenalidomide and dexamethasone in multiply relapsed/refractory myeloma patients; a phase I/II study. Br J Haematol. 2010;150:326-33.

42. Reece DE, Masih-Khan E, Atenafu EG, Jimenez-Zepeda VH, Anglin P, Chen C, et al. Phase I-II trial of oral cyclophosphamide, prednisone and lenalidomide for the treatment of patients with relapsed and refractory multiple myeloma. $\mathrm{Br} \mathrm{J}$ Haematol. 2015;168:46-54.

43. Cook G, Williams C, Brown JM, Cairns DA, Cavenagh J, Snowden JA, et al. Highdose chemotherapy plus autologous stem-cell transplantation as consolidation therapy in patients with relapsed multiple myeloma after previous autologous stem-cell transplantation (NCRI Myeloma X Relapse [Intensive trial]): a randomised, open-label, phase 3 trial. Lancet Oncol. 2014;15:874-85. 44. Cook G, Ashcroft AJ, Cairns DA, Williams CD, Brown JM, Cavenagh JD, et al. The effect of salvage autologous stem-cell transplantation on overall survival in patients with relapsed multiple myeloma (final results from BSBMT/UKMF Myeloma X Relapse [Intensive]): a randomised, open-label, phase 3 trial. The Lancet Haematology. 2016;3:e340-51.

45. Giralt S, Garderet L, Durie B, Cook G, Gahrton G, Bruno B, et al. American Society of Blood and Marrow Transplantation, European Society of Blood and Marrow Transplantation, Blood and Marrow Transplant Clinical Trials Network, and International Myeloma Working Group Consensus Conference on Salvage Hematopoietic Cell Transplantation in Patients with Relapsed Multiple Myeloma. Biol Blood Marrow Transplant. 2015;21:2039-51.

46. Shimoni A, Hardan I, Ayuk F, Schilling G, Atanackovic D, Zeller W, et al. Allogenic hematopoietic stem-cell transplantation with reduced-intensity conditioning in patients with refractory and recurrent multiple myeloma: longterm follow-up. Cancer. 2010;116:3621-30.

47. Patriarca F, Einsele H, Spina F, Bruno B, Isola M, Nozzoli C, et al. Allogeneic stem cell transplantation in multiple myeloma relapsed after autograft: a multicenter retrospective study based on donor availability. Biol Blood Marrow Transplant. 2012;18:617-26.

48. Auner HW, Szydlo R, van Biezen A, Iacobelli S, Gahrton G, Milpied N, et al. Reduced intensity-conditioned allogeneic stem cell transplantation for multiple myeloma relapsing or progressing after autologous transplantation: a study by the European Group for Blood and Marrow Transplantation. Bone Marrow Transplant. 2013;48:1395-400.

49. Harousseau JL, Dimopoulos MA, Wang M, Corso A, Chen C, Attal M, et al. Better quality of response to lenalidomide plus dexamethasone is associated with improved clinical outcomes in patients with relapsed or refractory multiple myeloma. Haematologica. 2010;95:1738-44.

50. Niesvizky R, Richardson PG, Rajkumar SV, Coleman M, Rosinol L, Sonneveld $P$, et al. The relationship between quality of response and clinical benefit for patients treated on the bortezomib arm of the international, randomized, phase 3 APEX trial in relapsed multiple myeloma. Br J Haematol. 2008;143:46-53. 
51. Dimopoulos MA, Kastritis E, Christoulas D, Migkou M, Gavriatopoulou M, Gkotzamanidou M, et al. Treatment of patients with relapsed/refractory multiple myeloma with lenalidomide and dexamethasone with or without bortezomib: prospective evaluation of the impact of cytogenetic abnormalities and of previous therapies. Leukemia. 2010;24:1769-78.

52. Sonneveld P, Avet-Loiseau H, Lonial S, Usmani S, Siegel D, Anderson KC, et al. Treatment of multiple myeloma with high-risk cytogenetics: a consensus of the International Myeloma Working Group. Blood. 2016;127:2955-62.

53. Lonial S, Vij R, Harousseau JL, Facon T, Moreau P, Mazumder A, et al. Elotuzumab in combination with lenalidomide and low-dose dexamethasone in relapsed or refractory multiple myeloma. J Clin Oncol. 2012;30:1953-9. 54. San-Miguel JF, Richardson PG, Gunther A, Sezer O, Siegel D, Blade J, et al. Phase Ib study of panobinostat and bortezomib in relapsed or relapsed and refractory multiple myeloma. J Clin Oncol. 2013;31:3696-703.

55. Richardson PG, Siegel DS, Vij R, Hofmeister CC, Baz R, Jagannath S, et al. Pomalidomide alone or in combination with low-dose dexamethasone in relapsed and refractory multiple myeloma: a randomized phase 2 study. Blood. 2014;123:1826-32.

56. Avet-Loiseau H. Ultra high-risk myeloma. Hematology Am Soc Hematol Educ Program. 2010;2010:489-93.

57. Moreau P, Cavo M, Sonneveld P, Rosinol L, Attal M, Pezzi A, et al. Combination of international scoring system 3 , high lactate dehydrogenase, and $t(4 ; 14)$ and/or del(17p) identifies patients with multiple myeloma (MM) treated with front-line autologous stem-cell transplantation at high risk of early MM progression-related death. J Clin Oncol. 2014;32:2173-80.

58. Chng WJ, Dispenzieri A, Chim CS, Fonseca R, Goldschmidt H, Lentzsch S, et al. IMWG consensus on risk stratification in multiple myeloma. Leukemia.

2014;28:269-77.

59. Morabito F, Gentile M, Ciolli S, Petrucci MT, Galimberti S, Mele G, et al. Safety and efficacy of bortezomib-based regimens for multiple myeloma patients with renal impairment: a retrospective study of Italian Myeloma Network GIMEMA. Eur J Haematol. 2010;84:223-8.

60. Richardson PG, Baz R, Wang M, Jakubowiak AJ, Laubach JP, Harvey RD, et al. Phase 1 study of twice-weekly ixazomib, an oral proteasome inhibitor, in relapsed/refractory multiple myeloma patients. Blood. 2014;124:1038-46. 61. Eriksson T, Hoglund P, Turesson I, Waage A, Don BR, Vu J, et al. Pharmacokinetics of thalidomide in patients with impaired renal function and while on and off dialysis. J Pharm Pharmacol. 2003;55:1701-6.

62. Weisel KC, Dimopoulos MA, Moreau P, Lacy MQ, Song KW, Delforge M, et al. Analysis of renal impairment in MM-003, a phase III study of pomalidomide + low - dose dexamethasone versus high - dose dexamethasone in refractory or relapsed and refractory multiple myeloma. Haematologica. 2016;101:872-8. 63. Zhong Y, Chen EY, Liu R, Chuang PY, Mallipattu SK, Tan CM, et al.

Renoprotective effect of combined inhibition of angiotensin-converting enzyme and histone deacetylase. J Am Soc Nephrol. 2013;24:801-11.

64. Advani A, Huang Q, Thai K, Advani SL, White KE, Kelly DJ, et al. Long-term administration of the histone deacetylase inhibitor vorinostat attenuates renal injury in experimental diabetes through an endothelial nitric oxide synthasedependent mechanism. Am J Pathol. 2011;178:2205-14. 
65. Berdeja J, Jagannath S, Zonder J, Badros A, Kaufman JL, Manges R, et al. Pharmacokinetics and Safety of Elotuzumab Combined With Lenalidomide and Dexamethasone in Patients With Multiple Myeloma and Various Levels of Renal Impairment: Results of a Phase Ib Study. Clin Lymphoma Myeloma Leuk. 2016;16:129-38.

66. Dimopoulos MA, Sonneveld P, Leung N, Merlini G, Ludwig H, Kastritis E, et al. International Myeloma Working Group Recommendations for the Diagnosis and Management of Myeloma-Related Renal Impairment. J Clin Oncol. 2016;34:154457.

67. San Miguel JF, Lahuerta JJ, Garcia-Sanz R, Alegre A, Blade J, Martinez R, et al. Are myeloma patients with renal failure candidates for autologous stem cell transplantation? Hematol J. 2000;1:28-36.

68. Leebeek FW. Update of thrombosis in multiple myeloma. Thromb Res. 2016;140 Suppl 1:S76-80.

69. Terpos E, Kleber M, Engelhardt M, Zweegman S, Gay F, Kastritis E, et al. European Myeloma Network guidelines for the management of multiple myeloma-related complications. Haematologica. 2015;100:1254-66.

70. Kumar S, Mahmood ST, Lacy MQ, Dispenzieri A, Hayman SR, Buadi FK, et al. Impact of early relapse after auto-SCT for multiple myeloma. Bone Marrow Transplant. 2008;42:413-20. 
Figure legend.

Figure 1. Principal regimen available for treatment of first relapse after autologous stem cell transplantation (ASCT) and relevant factors for treatment selection. allo-SCT is for allogeneic stem cell transplantation. 
Treatment MM relapse after ASCT

Table 1. Overview of recent studies investigating newer agent combinations in relapsed multiple myeloma

\begin{tabular}{|c|c|c|c|c|c|c|c|c|c|}
\hline Study & Phase & $\mathrm{N}$ & Regimen & $\begin{array}{l}\text { Prior } \\
\text { lines }\end{array}$ & $\begin{array}{l}\text { ORR } \\
\%\end{array}$ & $\begin{array}{l}\geq \mathrm{CR} \\
\%\end{array}$ & $\begin{array}{l}\text { PFS } \\
\mathrm{m}\end{array}$ & $\begin{array}{l}\mathrm{OS} \\
\mathrm{m}\end{array}$ & $\begin{array}{l}\text { Median } \\
\text { FU } \\
\mathrm{m}\end{array}$ \\
\hline Dimopoulos et al. 2007[21] & III & 176 & $\mathrm{Rd}$ & $\geq 1$ & 60.2 & 15.9 & 11.3 & NT & 16.4 \\
\hline MM-010 & & 175 & $\mathrm{D}$ & & 24.0 & 3.4 & 4.7 & 20.6 & \\
\hline Weber et al. 2007[22] & III & 177 & $\mathrm{Rd}$ & $\geq 1$ & 61.0 & 14.1 & 11.1 & 29.6 & 17.6 \\
\hline MM-009 & & 176 & $\mathrm{D}$ & & 19.9 & 0.6 & 4.7 & 20.2 & \\
\hline Stewart et al. 2015 & III & 396 & CFZ-Rd & $2(1-3)$ & 87.1 & 31.8 & 26.3 & NR & 32.3 \\
\hline ASPIRE[24] & & 396 & $\mathrm{Rd}$ & & 66.7 & 9.3 & 17.6 & NR & 31.5 \\
\hline Dimopoulos et al. 2016 & III & 464 & CFZ-d & $2(1-2)$ & 77 & 13 & 18.7 & NR & 12.5 \\
\hline ENDEAVOR[27] & & 465 & BTZ-d & & & 6 & 9.4 & NR & 11.9 \\
\hline Moreau et al. 2016 & III & 360 & IXA-Rd & $1(1-3)$ & 78.3 & 12 & 20.6 & NR & 23.3 \\
\hline TOURMALINE-MM1[29] & & 362 & $\mathrm{Rd}$ & & 71.5 & 7 & 14.7 & NR & 22.9 \\
\hline Lonial et al. 2015 & III & 321 & ELO-Rd & $2(1-4)$ & 79 & 4 & 19.4 & NR & 24.5 \\
\hline ELOQUENT-2[31] & & 325 & $\mathrm{Rd}$ & & 66 & 7 & 14.9 & NR & \\
\hline \multirow[t]{2}{*}{ Jakubowiak et al. 2016[32] } & II & 77 & ELO-BTZ-d & $1(1-3)$ & 66 & 4 & 9.7 & NR & 15.9 \\
\hline & & 75 & BTZ-d & $1(1-3)$ & 63 & 3 & 6.9 & NR & 11.7 \\
\hline Dimopoulos et al. 2016 & III & 286 & DARA-Rd & $1(1-11)$ & 93 & 43 & NR & NR & 13.5 \\
\hline POLLUX[34] & & 283 & $\mathrm{Rd}$ & $1(1-8)$ & 76 & 19 & 18.4 & NR & \\
\hline Palumbo et al. 2016 & III & 251 & DARA-BTZ-d & $2(1-\geq 4)$ & 83 & 19 & NR & NR & 7.4 \\
\hline CASTOR[35] & & & BTZ-d & $2(1-\geq 4)$ & 63 & 9 & 7.2 & NR & \\
\hline San-Miguel et al. 2014 & III & 387 & PAN-Vd & $1(1-3)$ & 60.7 & 11 & 11.99 & 33.6 & 6.5 \\
\hline
\end{tabular}




\section{ACCEPTED MANUSCRIPT}

Treatment MM relapse after ASCT

\begin{tabular}{|c|c|c|c|c|c|c|c|c|c|}
\hline PANORAMA-1[18] & & 381 & $\mathrm{Vd}$ & & 54.6 & 6 & 8.08 & 30.4 & 5.6 \\
\hline Dimopoulos et al. 2013 & III & 317 & VOR-Vd & $2(1-3)$ & 56 & 8 & 7.63 & NR & 14.2 \\
\hline VANTAGE-088[19] & & 320 & Vd & & 41 & 5 & 6.83 & & \\
\hline San Miguel et al. 2013 & III & 302 & POM-d & $5(2-14)$ & 31 & 1 & & 11.9 & 10 \\
\hline MM-003[38] & & 153 & $\mathrm{D}$ & $5(2-17)$ & 10 & 0 & & 7.8 & \\
\hline Cook et al. 2014 & III & 89 & HDM-ASCT & 1 & 83 & 39 & 19 & 67 & 50 \\
\hline NCRI Myeloma X Relapse[43, 44] & & 85 & Сy & & 75 & 22 & 11 & 52 & 54 \\
\hline
\end{tabular}

Abbreviations: ORR, overall response rate; CR, complete remission; PFS, progression free survival; m, month; OS, overall survival; FU, follow-up; Rd, lenalidomide-dexamethasone; D, high dose dexamethasone, CFZ, carfilzomib; IXA, ixazomib; ELO, elotuzumab; DARA, daratumumab; PAN, panobinostat; Vd, bortezomib-dexamethasone; VOR, vorinostat; POM, pomalidomide. 


\section{ACCEPTED MANUSCRIPT}

\section{ASCT (melphalan 200) based frontline treatment}

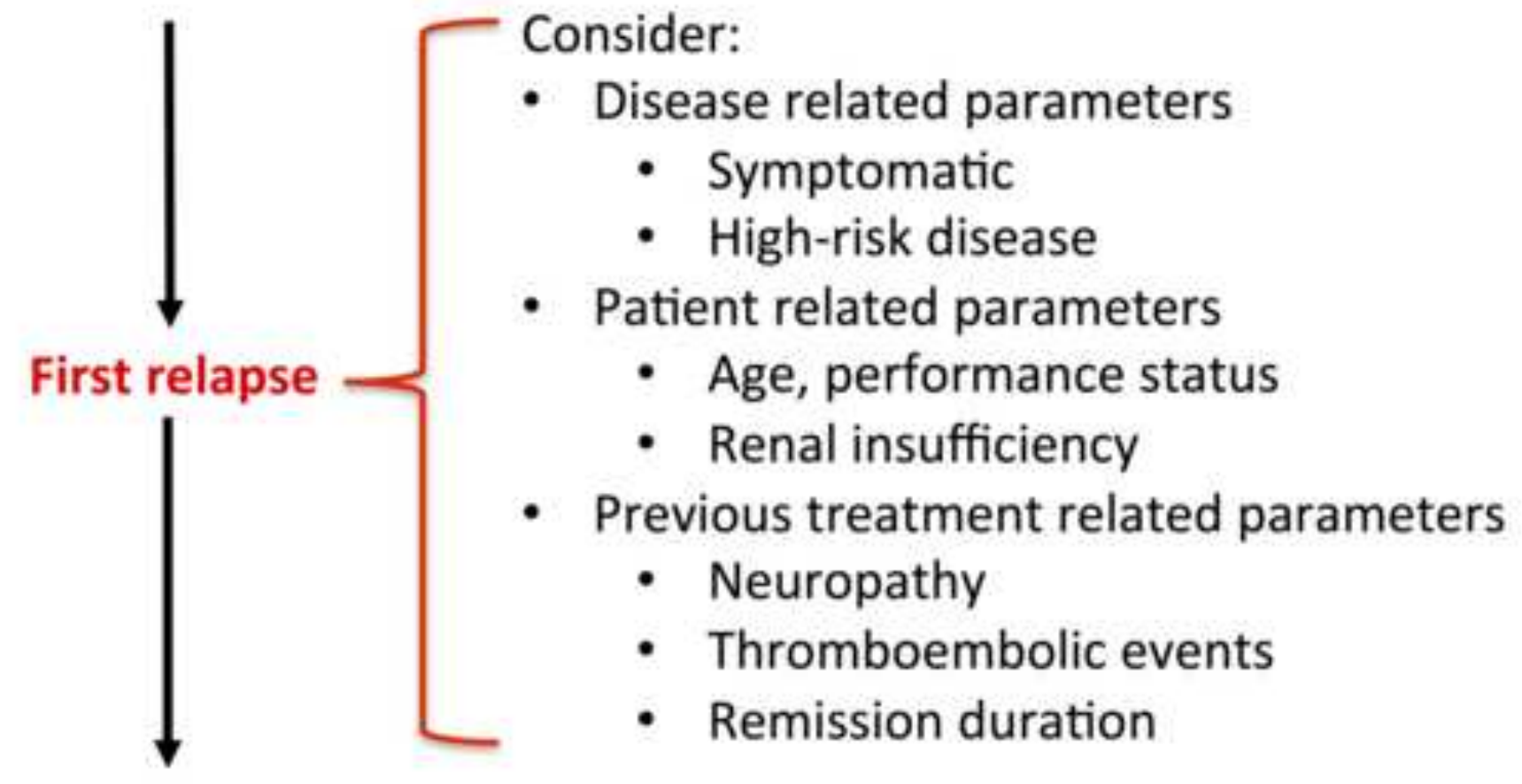

\section{Bortezomib + dexamethasone in combination with:}

- Cyclophosphamide

- Pegylated doxorubicin

- Thalidomide or lenalidomide

- Panobinostat

- Daratumumab

\section{Lenalidomide + dexamethasone in combination with:}

- Cyclophosphamide

- Carfilzomib

- Ixazomib

- Elotuzumab

- Daratumumab

\section{others}

- carfilzomib + dexamethasone

- pomalidomide + dexamethasone

- second ASCT, allo-SCT 
Treatment MM relapse after ASCT

\section{Highlights}

- No standard of care for multiple myeloma relapse after autologous transplantation

- Combination regimen including one or two novels agents are generally preferred

- Treatment is individualized based on toxicity, patient and disease characteristics 